\title{
Point-to-point Coulomb effects in high brightness photoelectron beam lines for ultrafast electron diffraction
}

\author{
M. Gordon $\odot,{ }^{1}$ S. B. van der Geer $\odot,{ }^{2}$ J. Maxson, ${ }^{3}$ and Y.-K. Kim $\oplus^{1}$ \\ ${ }^{1}$ University of Chicago Department of Physics, Chicago, Illinois 60637, USA \\ ${ }^{2}$ Pulsar Physics, Burghstraat 47, 5614 BC Eindhoven, The Netherlands \\ ${ }^{3}$ Cornell University Department of Physics, Ithaca, New York 14853, USA
}

(Received 20 April 2021; accepted 2 August 2021; published 24 August 2021)

\begin{abstract}
In an effort to increase spatial and temporal resolution of ultrafast electron diffraction and microscopy, ultrahigh-brightness photocathodes are actively sought to improve electron beam quality. Beam dynamics codes often approximate the Coulomb interaction with mean-field space charge, which is a good approximation in traditional beams. However, point-to-point Coulomb effects, such as disorder-induced heating (DIH) and the Boersch effect, cannot be neglected in cold, dense beams produced by such photocathodes. In this paper, we introduce two new numerical methods to calculate the important effects of the photocathode image charge when using a point-to-point interaction model. Equipped with an accurate model of the image charge, we calculate the effects of point-to-point interactions on two high-brightness photoemission beam lines for ultrafast diffraction. The first beam line uses a $200 \mathrm{keV}$ gun, whereas the second uses a $5 \mathrm{meV}$ gun, each operating in the single-shot diffraction regime with $10^{5}$ electrons/pulse. For the beam lines simulated in this paper, assuming a zero photoemission temperature, it is shown that including stochastic Coulomb effects increases the final emittance by over a factor of 2 and decreases the peak transverse phase space density by over a factor of 3 as compared to mean-field simulations. We then introduce a method to compute the energy released by DIH using the pair correlation function and approximate the contribution DIH has on the emittance, which may serve as a reasonable estimate for the effects of DIH beyond the cases studied in this work. This DIH energy was found to scale very near the theoretical result for stationary ultracold plasmas, and it accounts for over half of the emittance growth above mean-field simulations.
\end{abstract}

DOI: $10.1103 /$ PhysRevAccelBeams.24.084202

\section{INTRODUCTION}

The development of high-brightness photocathodes is a driving force in the improvement of electron accelerator technologies such as free electron lasers, energy recovery linacs, and ultrafast electron diffraction (UED) and microscopy. The brightness of the beams used in these applications is set at the electron source and can only degrade during further acceleration and transport. Consequently, the brightness of the electron source defines the ultimate limits of the capabilities of these devices [1-4]. The photocathode brightness is set by two parameters: the density of electrons emitted from the source, and their mean transverse energy (MTE), which acts as an effective beam temperature [5,6]. Increasing the electron density at the source is not always a viable option, as space charge forces can reduce brightness downstream dramatically. While some of this brightness

Published by the American Physical Society under the terms of the Creative Commons Attribution 4.0 International license. Further distribution of this work must maintain attribution to the author(s) and the published article's title, journal citation, and DOI. can be restored via emittance compensation, some is lost to nonlinear distortions which are challenging to reverse $[7,8]$. However, it has been shown in many modern applications that reducing the MTE of the photocathode can still lead to large gains in brightness [9].

Reducing the MTE of photocathodes is a very active area of research in which significant progress has been made in the last decade. Typical photocathodes used in accelerator facilities today have a MTE of a few hundred meV [10-12] whereas near threshold emission at room temperature has demonstrated electron beams with an MTE of $\sim 25 \mathrm{meV}$ [13]. Furthermore, cryocooled photocathodes near threshold have shown the capability to go down even further to an MTE of $\sim 5 \mathrm{meV}$ [14]. At these low temperatures, pointto-point interactions play an increasingly important role in the overall beam dynamics, as shown by the following argument.

The mean-field approximation commonly used in simulation codes is only valid when there are many particles in a Debye sphere. This Debye screening length is given by

$$
\lambda=\sqrt{\frac{\epsilon_{0} k T}{n_{0} e^{2}}}
$$


where $\epsilon_{0}$ is the permittivity of free space, $\mathrm{k}$ is the Boltzmann constant, $T$ is the temperature of the beam, $n_{0}$ is the volume number density of the beam, and $e$ is the charge of the electron. For $k T=5 \mathrm{meV}$ and a density of $10^{17} \mathrm{~m}^{-1 / 3}$ (commonly achieved in photoinjectors today), the Debye screening length is approximately $1.7 \mu \mathrm{m}$. However, the average interparticle spacing $n_{0}^{-1 / 3}$ at the same density is $2.2 \mu \mathrm{m}$. Thus, very few electrons will be within one Debye screening length of any given electron [15]. This situation has been studied extensively for ultracold gas-based plasma and electron sources, which exhibit single meV electron temperatures in photoemission [16-23].

Brute force calculation of the pairwise Coulomb interaction scales with the square of the number of electrons, $\mathcal{O}\left(N^{2}\right)$, making it prohibitively time-consuming to exactly simulate dynamics with large number of electrons. Thus, to accurately capture the beam dynamics, approximation methods are used which compute pairwise interactions of nearby particles, while approximating long-range interactions using the mean-field approach. These methods scale as $\mathcal{O}(N \log N)$ for traditional tree-based methods and $\mathcal{O}(N)$ for the fast multipole method, making them feasible for simulation [24,25]. We will refer to these methods as point-to-point methods.

A critical challenge in employing a classical pointcharge force model for a photoelectron source is the unphysical divergence of the image potential at the cathode surface. The underlying cause of the problem is that classical equations of motion are not valid at and very near the cathode surface. In a classical simulation, however, the size of the integration step typically scales inversely proportional to the gradient of the potential. Thus, near a divergence, the integration step can limit to zero. This produces a scale-matching problem wherein very small step sizes must be maintained throughout the particle emission process, which can lead to prohibitively long simulation times. However, as will be shown below, image charge effects significantly impact beam size and emittance evolution, and cannot be ignored.

This work aims to extend the work on Coulomb effects in ultracold plasma electron sources to photocathode guns, which can potentially support even higher beam density. We provide a new method to compute the image force which is free of divergences and tuning parameters. Using this model of the overall beam dynamics, we turn to introduce new microscopic figures of merit to disentangle the global and local effects of point-to-point interactions.

To show the generality of the new methods, we examine beam dynamics in two very different UED beam lines based on archetypes used in practice today: a $200 \mathrm{keV}$ dc gun with lower total initial beam density $\left(\sim 10^{17} \mathrm{~m}^{-3}\right)$, and a high gradient $5 \mathrm{MeV}$ rf photoinjector with higher initial beam density $\left(\sim 10^{18} \mathrm{~m}^{-3}\right)$. UED is a good test case for examining point-to-point effects as the number of particles needed per bunch is often relatively small $\left(10^{5}-10^{7}\right)$ in comparison to synchrotron radiation applications, making it feasible to simulate every particle in the bunch with modest computing resources. Along with this, the short bunch lengths, small spot sizes, and long coherence lengths needed to make atomic scale resolution diffraction patterns with femtosecond time resolution ultimately result in peak current densities comparable to those in free electron laser injectors $[1,26]$.

Using our new method of calculating near-photocathode dynamics, we highlight one unique point-to-point phenomenon called disorder-induced heating (DIH) which arises very near the photocathode. DIH was originally studied in the ultracold plasma community (see, e.g., Ref. [27]), but it may have significant implications for cold photoemitted electron beams [20,28]. DIH is the thermalization of the initial potential energy stored in the random positions of near neighbor photoelectrons. Upon thermalization, the particle distribution develops a characteristic microscopic structure with a lack of near neighbors, and the beam simultaneously suffers emittance growth due to the increased temperature. For a stationary (nonaccelerating, no expansion) electron bunch starting with zero temperature, the temperature rise due to $\mathrm{DIH}$ is given by

$$
k T_{\mathrm{DIH}}=\frac{C e^{2}}{4 \pi \epsilon_{0} a}
$$

where $a=\left(3 / 4 \pi n_{0}\right)^{1 / 3}$ is the Wigner-Seitz radius of the bunch and $k$ is the Boltzmann constant. $C$ is a dimensionless constant which can be determined by tabulated plasma correlation energies to be roughly $C \approx 0.45[28,29]$. The timescale $\tau$ of the thermalization can be calculated to be a constant fraction of a plasma period [30]. In photoinjectors, this time is typically of the order of ten picoseconds (ps) or less, resulting in thermalization near the photocathode during initial acceleration. A correct image charge model is thus critical to understand DIH in photoinjectors. Furthermore, the beam density can change significantly near the photocathode due to space charge expansion and acceleration. The resulting balance has not been studied in detail in photocathode guns before.

In this work, we introduce new methods for simulating high-brightness photoelectron beam lines. We simulate two UED beam lines with $0 \mathrm{meV}$ MTE with multiple methods of calculating the electrons interactions to show why these new methods are crucial for accurately determining the capabilities of these devices. Next, we quantify DIH via an analysis of the resulting microscopic density distribution. We then estimate the rms emittance increase attributable to $\mathrm{DIH}$ and find that it is the dominant source of emittance dilution in the two cases under study.

\section{POINT-TO-POINT SIMULATION METHODS}

\section{A. Coulomb interactions and image charge model}

The simulations shown in this paper were performed with the space charge tracking code General Particle 
Tracer (GPT) [31] using three different algorithms. The first is GPT's mean-field space charge algorithm, a nonequidistant 3D multigrid Poisson solver [32], which is used to calculate the mean-field interaction of the entire bunch, including image charge effects. The second is the Barnes-Hut algorithm internal to GPT [24], with a Barnes-Hut angle parameter of 0.3, used to model point-to-point Coulomb interactions. It does this by exactly computing the electric fields from near neighbor particles at each time step using Coulomb's law whereas long-range interactions are approximated using multipole expansions. This makes the Barnes-Hut algorithm capable of simulating stochastic effects like DIH and Boersch effect, while also taking into account long-range space charge forces. However, due to divergent fields at the cathode, the Barnes-Hut algorithm does not allow for simple inclusion of image effects. To include these effects, we developed a third technique which we call the plus-minus-plus (PMP) Method.

The PMP method approximates the image charge as arising from a mean-field calculation and the total space charge force is calculated in a three step process as depicted in Fig. 1. In PMP, GPT's mean-field space charge algorithm is used to calculate the mean-field interaction of the entire bunch, including image effects. Subsequently, a second call is made to the mean-field solver, but this time without the cathode boundary condition. By subtracting this field from the initial full mean-field space charge calculation, the mean-field approximation of the image field alone is extracted. The last step in the PMP process adds the stochastic interactions to the previously obtained meanfield image charges with the stochastic Barnes-Hut pointto-point method.

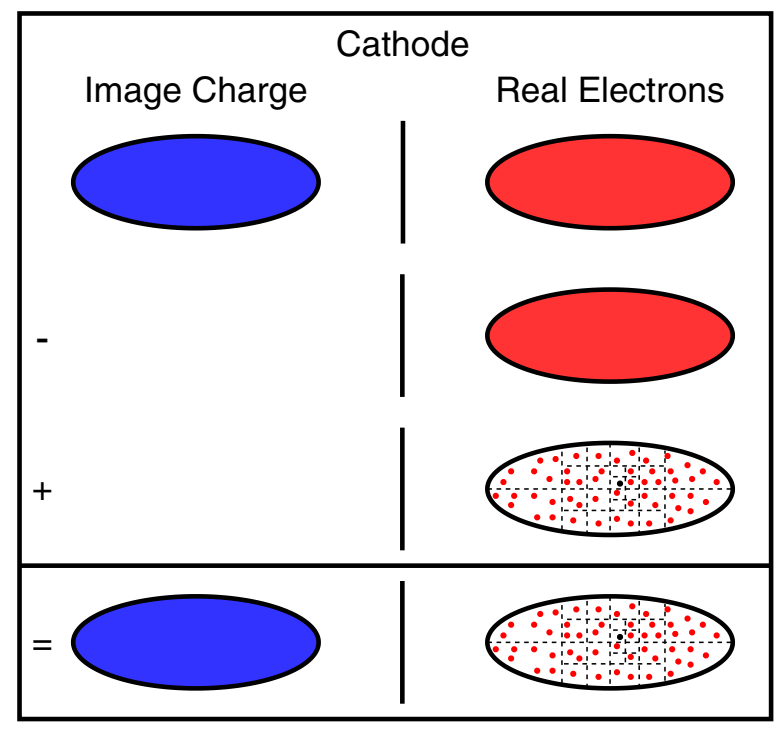

FIG. 1. Depiction of PMP three-step space charge calculation. Filled in ellipses represent mean-field calculation of electric fields, and ellipses filled with dots represent a Barnes-Hut calculation of electric fields.
In an Appendix, we discuss the accuracy of the assumption of a mean-field image force. Specifically, we compare the PMP method with another image charge method which includes point-to-point effects via a dynamical image charge potential which does not diverge [33]. The latter requires additional computing time and tuning parameters, and in general we find good agreement with PMP. Thus, PMP is our method of choice throughout.

To achieve an accurate accounting of stochastic Coulomb effects, each macroparticle represents exactly one electron in all simulations, and all distributions are pseduorandom, rather than quasirandom. These same macroparticle settings were used in the mean-field simulations for consistency.

To test the convergence of GPT's mean-field space charge algorithm, the same beam line was simulated multiple times using different space charge solver parameters. We set a convergence limit of 5\% relative variation in rms quantities with increasing space charge accuracy. We found that nearly all default settings for GPT's mean field space charge solver were sufficient to satisfy this limit, except for the total number of meshlines which was increased by $20 \%$ such that the variation was below this threshold.

\section{B. Using $90 \%$ rms figures of merit}

Root-mean-squared (rms) figures of merit for beam size/ length, energy spread, and emittance are not well defined in the presence of strong point-to-point Coulomb interactions. This is due to the presence of large angle scattering which generates long-tailed distributions for which rms values diverge [34]. To avoid sensitivity to outliers, but retain the sense of the traditional accelerator figures of merit, all quantities presented in this paper are calculated using $90 \%$ rms values unless otherwise denoted, wherein a subset of the distribution containing $90 \%$ of the particles are chosen such that the metric in question is minimized.

\section{DESCRIPTION OF DC AND NCRF GUN UED BEAM LINES}

Both beam line designs considered here originated from a multiobjective genetic algorithm optimization study, using the mean-field space charge model, to provide an emittance minimum at the sample plane with realistic

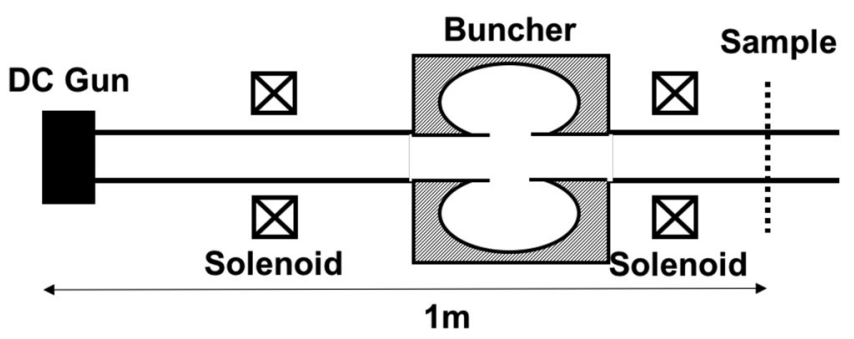

FIG. 2. Layout of the cryocooled dc gun UED beam line used in the following simulations. 
TABLE I. dc beam line simulation beam parameters.

\begin{tabular}{lc}
\hline \hline Parameter & Value \\
\hline Bunch charge (fC) & -14 \\
Transverse rms size $(\mu \mathrm{m})$ & 8.1 \\
Laser pulse length $(\mathrm{ps})$ & 9.8 \\
\hline \hline
\end{tabular}

constraints on the bunch length and spot size. These optimizations are described in Refs. [9,35,36]. Each case shown here is an individual from a multiobjective genetic algorithm Pareto optimal frontier with a MTE of $0 \mathrm{meV}$. Individuals were selected containing a charge closest to $10^{5}$ electrons/pulse and were then reevalutated using the PMP method.

The lower energy dc beam line, depicted schematically in Fig. 2, consists of a cryocooled $200 \mathrm{kV}$ dc gun [37] with an extraction electric field of $11.25 \mathrm{MV} / \mathrm{m}$, followed by a solenoid, a normal conducting $3.0 \mathrm{GHz}$ buncher cavity of the Eindhoven design [38], and a second solenoid. The beam line was optimized to have a minimal emittance at a sample location approximately $1 \mathrm{~m}$ from the cathode when emitting an electron beam with $0 \mathrm{meV}$ MTE [35]. Beam parameters are shown in Table I.

The higher energy normal conducting radio frequency (NCRF) beam line, depicted schematically in Fig. 3, consists of a 1.6 cell $2.856 \mathrm{GHz} \mathrm{NCRF}$ gun of the BNL/SLAC/UCLA design [39], with a peak electric field of $100 \mathrm{MV} / \mathrm{m}$, launch phase of $38.6^{\circ}$ from peak field, and final beam energy of $5 \mathrm{MeV}$, followed by a solenoid, a 9 cell buncher cavity, and a second solenoid. The buncher is modeled using nine copies of the first cell of the SLAC linac [40]. The beam line was optimized to have a minimal emittance at a sample location approximately $2.5 \mathrm{~m}$ from the cathode when emitting an electron beam with $0 \mathrm{meV}$ MTE [36]. Beam parameters are shown in Table II.

\section{MACROSCOPIC BEAM EVOLUTION}

The $90 \%$ rms transverse size of the beam along the dc and NCRF UED beam line is shown in Figs. 4(a) and 5(a), respectively. In all simulations, space charge increases the beam size, after which the first solenoid matches the beam size into the buncher cavity (which has a noticeable transverse defocusing), and the second solenoid forms the final waist. As expected from the emittance compensation

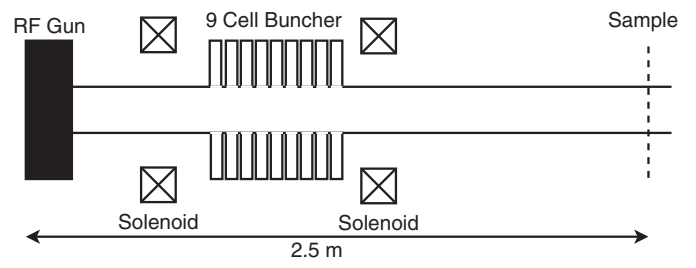

FIG. 3. Layout of the NCRF gun beam line used in the following simulations.
TABLE II. NCRF beam line simulation beam parameters.

\begin{tabular}{lc}
\hline \hline Parameter & Value \\
\hline Bunch charge (fC) & -17 \\
Transverse rms size $(\mu \mathrm{m})$ & 2.5 \\
Laser pulse length $(\mathrm{ps})$ & 3.2 \\
\hline \hline
\end{tabular}

process, the emittance minimum occurs very near the beam size waist.

In the Barnes-Hut simulation without the cathode boundary condition, the initial space charge blowup leads to a larger spot size at the first solenoid. Because of this, the focusing elements cause the beam waist to occur earlier than when the image force is included. Thus, omission of the image force generates significantly different beam dynamics.

The difference between the mean-field and PMP simulation is solely due to point-to-point effects, as a mean-field image force is included in each. It is interesting to note that

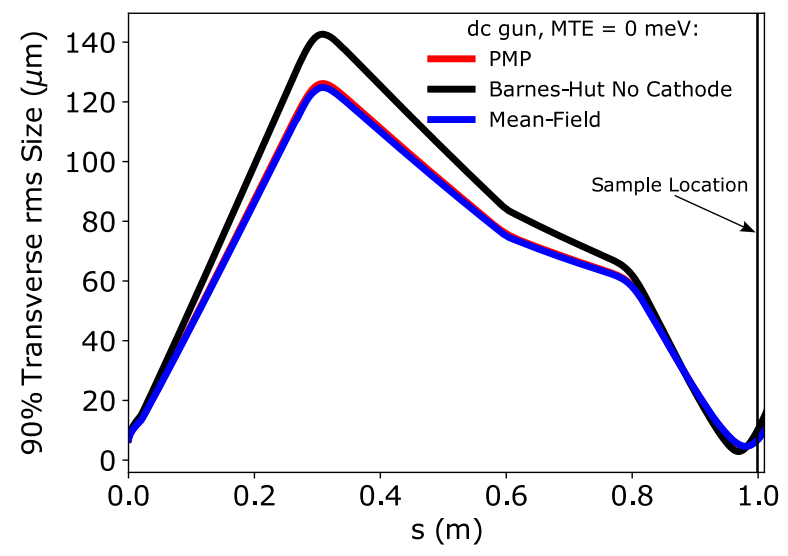

(a)

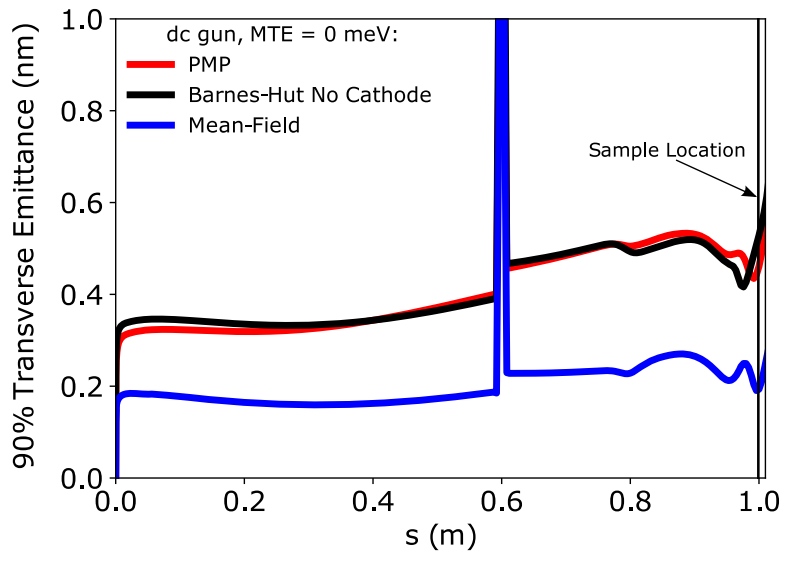

(b)

FIG. 4. (a) Spot size and (b) transverse normalized rms emittance comparison between the PMP method, Barnes-Hut method without a cathode, and mean-field space charge simulations of the dc UED beam line with $0 \mathrm{meV}$ MTE. 


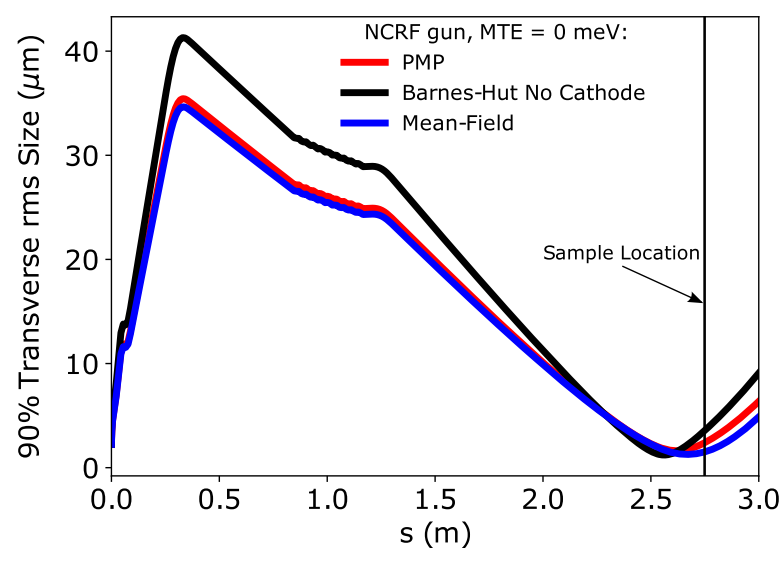

(a)

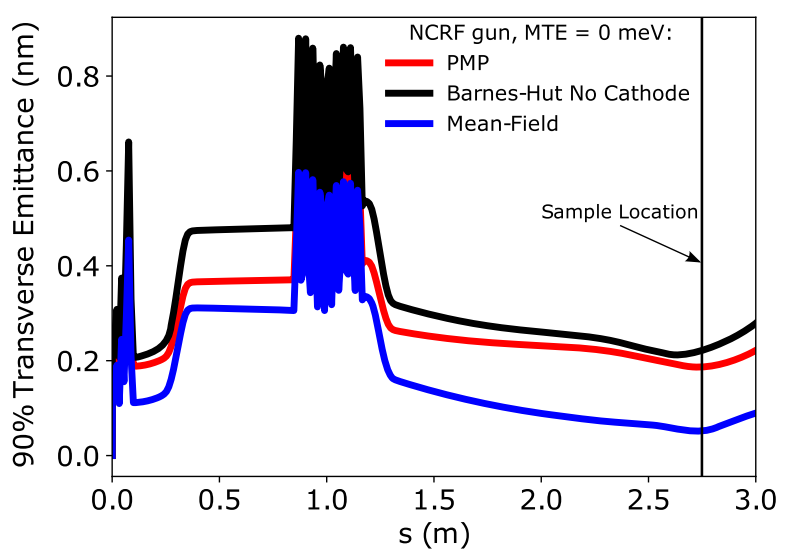

(b)

FIG. 5. (a) Spot size and (b) transverse normalized rms emittance comparison between the PMP method, Barnes-Hut method without a cathode, and mean-field space charge simulations of the NCRF UED beamline with $0 \mathrm{meV}$ MTE.

the slightly larger spot size in PMP simulations translates to noticeably stronger focusing downstream. In the dc gun beam line, the stronger focusing is noted by a smaller beam size at the focus, and in the NCRF beam line, the beam waist is formed earlier by $5.5 \mathrm{~cm}$.

The evolution of the normalized transverse rms emittance for the dc and NCRF UED beam line with $0 \mathrm{meV}$ MTE is shown in Figs. 4(b) and 5(b), respectively. Starting at an emittance of zero, the emittance quickly grows as the beams experience both the mean-field space charge phase space shearing, and also a growth in temperature due to the thermalization of the initial stochastic potential energy stored between near neighbors.

Comparing the transverse $90 \%$ rms emittance of the Barnes-Hut method without a cathode to the PMP method, we find that qualitatively they behave similarly. However, due to the earlier location of the beam waist in the BarnesHut simulations, the location of the emittance minima also shifts to an earlier position.
Although at a higher beam energy, we note the NCRF beam line exhibits a larger relative effect from point-topoint interactions. Later, we will show that this can be explained by the effects of DIH with a larger initial electron number density. Though the space charge forces are more heavily suppressed at high energy, the effects of DIH thermalization occur at low energy near the cathode where relativistic suppression is negligible. The timescale of the evolution of the thermalization is a constant fraction of the plasma period [28], which in this case is roughly $30 \mathrm{ps.}$ The value of the emittance is over a factor of 3.7 larger than when using only the mean-field approximation when including point-to-point effects.

It should be noted that because these beam lines were optimized to minimize the emittance of the mean-field space charge beam with $0 \mathrm{meV}$ MTE at the sample, the emittance in the PMP simulation is not necessarily optimized to be maximally compensated at its respective minimum. Thus, these numbers represent an upper bound to the maximal effect of point-to-point space charge on the emittance for these UED beam lines. However, as we will show later, most of the emittance growth above the meanfield case arises from microscopic DIH-like effects, which are insensitive to small perturbations in the focusing optics.

The slice energy spread results from the two beam lines are shown in Table III. In both beam lines, the slice energy spread slowly varies except for jumps at their respective gun, buncher, and beam waist. The energy spread has time dependence affecting the second significant figure, so only the most significant figure is reported.

In all simulations, the bunch length is approximately constant until the buncher, after which it decreases linearly in time. In the dc beam line, the bunch length at the sample is $0.92 \mathrm{ps}$ in the PMP simulation and $0.91 \mathrm{ps}$ in the meanfield simulation. In the NCRF beam line, the bunch length at the sample is $0.91 \mathrm{ps}$ in both simulations. Thus, point-topoint effects do not play a large role in determining bunch length at the sample. We note here that the bunch lengths at the sample location are higher here than in many UED setups which achieve temporal resolution well below 1 ps. Optimizations of time-resolved electron scattering instruments inherently have a tradeoff between transverse emittance and longitudinal size, and the optimal point on this tradeoff curve depends on the phenomenon being studied.

TABLE III. Slice energy spread in $0 \mathrm{meV}$ MTE simulations.

\begin{tabular}{lcc}
\hline \hline Simulation & $\frac{\sigma_{E}}{\bar{E}}$ before buncher & $\frac{\sigma_{E}}{\bar{E}}$ after buncher \\
\hline dc(PMP) & $5 \times 10^{-6}$ & $5 \times 10^{-5}$ \\
dc(Barnes-Hut) & $5 \times 10^{-6}$ & $5 \times 10^{-5}$ \\
dc(mean-field) & $9 \times 10^{-7}$ & $5 \times 10^{-5}$ \\
NCRF(PMP) & $4 \times 10^{-5}$ & $3 \times 10^{-4}$ \\
NCRF(Barnes-Hut) & $4 \times 10^{-5}$ & $3 \times 10^{-4}$ \\
NCRF(mean-field) & $4 \times 10^{-5}$ & $3 \times 10^{-4}$ \\
\hline \hline
\end{tabular}


By choosing a very small transverse emittance at the expense of bunch length, the longitudinal dynamics are less sensitive to stochastic effects than the transverse dynamics, making these simulations a more stringent test of DIH.

\section{MICROSCOPIC EVOLUTION}

We now move to analyze the microscopic real and phase space distributions in an effort to determine to what extent they follow simple predictions of beam heating via DIH, and to what extent this heating determines the total rms emittance growth from point-to-point effects.

\section{A. Core emittance}

One tool which can be used to analyze the microscopic evolution of a beam is the core emittance. The core emittance is a measure of the peak transverse phase space density. It is defined through an emittance vs particle fraction curve [41]. Starting with the full beam emittance (particle fraction of 1), particles can be excluded from the emittance calculation such that the resulting emittance is minimized, see Fig. 6. The core emittance is defined as the limit of the slope of the emittance vs particle fraction curve as the particle fraction goes to 0 . It is inversely proportional to the peak transverse phase space density,

$$
\epsilon_{c}=\left.\frac{d \epsilon}{d f}\right|_{f \rightarrow 0^{+}}=\frac{1}{4 \pi \rho_{0}},
$$

where $\epsilon$ is the emittance of the beam for a given fraction of particles, $f$ is the particle fraction, and $\rho_{0}$ is the peak phase space density. We expect $\rho_{0}$ to be invariant in mean-field space charge systems but the introduction of point-to-point effects can break this invariance. However, because we compute the core emittance with a finite number of beam

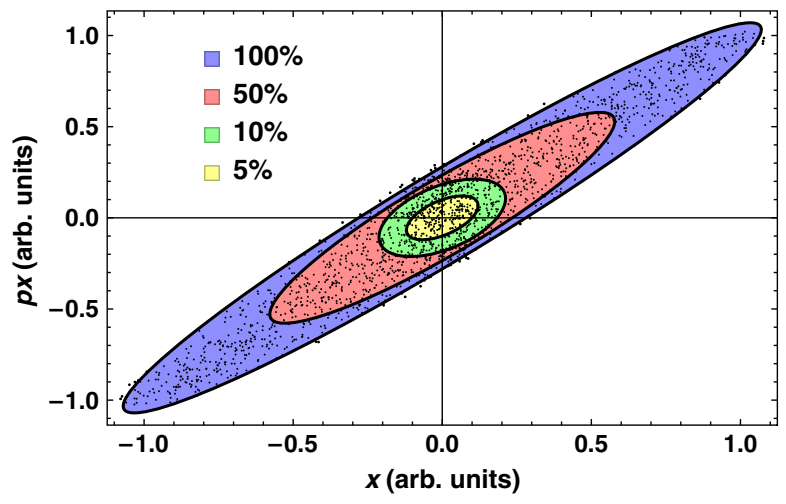

FIG. 6. Depiction of emittance vs particle fraction selection. Ellipses are drawn such that they represent the phase space area occupied by the beam using only a given fraction of the total number of particles. Ellipse dimensions are selected such that the emittance is minimized for each particle fraction. particles and a finite number of bounding ellipses the value will never be exactly zero even if we start with zero MTE.

In Figs. 7(a) and 7(b), the emittance vs fraction curves at the emittance minima are shown for mean-field and pointto-point space charge for the dc gun and NCRF gun UED beam lines. Note the sharp increase in rms emittance due to outliers when the particle fraction approaches unity. As can be seen, for small particle fractions, the emittance of the PMP simulation is significantly higher than that of the mean-field simulation. This shows that point-to-point effects not only have created more outliers but have fundamentally degraded beam quality up to and including the core of the beam, as would be expected from DIH. To help illustrate this further, phase space portraits are shown at the respective emittance minima for the two beam lines with mean-field space charge in Figs. 7(c) and 7(d) and with PMP space charge in Figs. 7(e) and 7(f). The decrease in core phase space density is clearly seen by the increased width in the $\gamma \beta_{x}$ coordinate in Figs. 7(e) and 7(f). Note that the faint diagonal tails in these figures are not outliers due to stochastic interactions but the effect of slightly mistuned optics.

The core emittance of the beam was computed at different points along the dc beam line, see Figs. 8 and 9 for the NCRF gun beam line. After a quick initial rise at low energy, the core emittance in the point-to-point simulations remains far above that of the mean-field simulation.

The core emittance at the sample of these simulations is shown in Table IV. As with the transverse rms emittance, the effects of point-to-point space charge are more distinct in the NCRF beam line, where the number density of electrons is higher.

\section{B. Radial distribution function}

The radial distribution function, $g(r)$, of a system of particles relates the bulk density of particles to the local particle density as a function of distance from a reference particle [42]. A microscopically uniform distribution of particles has a constant radial distribution function excluding effects from finite system size. In this case, a neighbor particle to a given reference particle is equally likely to be found at any distance. However, due to the divergence of the Coulomb interaction, the number of very near neighbors to a reference particle in an electron beam evolves to become zero. This is known as the Coulomb hole and it results in a decrease of the total potential energy of the system [43]. This release of potential energy causes DIH and we denote the resulting mean kinetic energy each particle gains from this heating by $E_{\mathrm{DIH}}$.

To calculate $g(r)$, the following procedure is used: screen-based outputs are taken from chosen positions along the beam line. Because we are interested in the dynamics of the core of the beam, only the $10 \%$ of electrons which are closest to the longitudinal center of the beam are used. For each of these particles, the distance to every other selected 


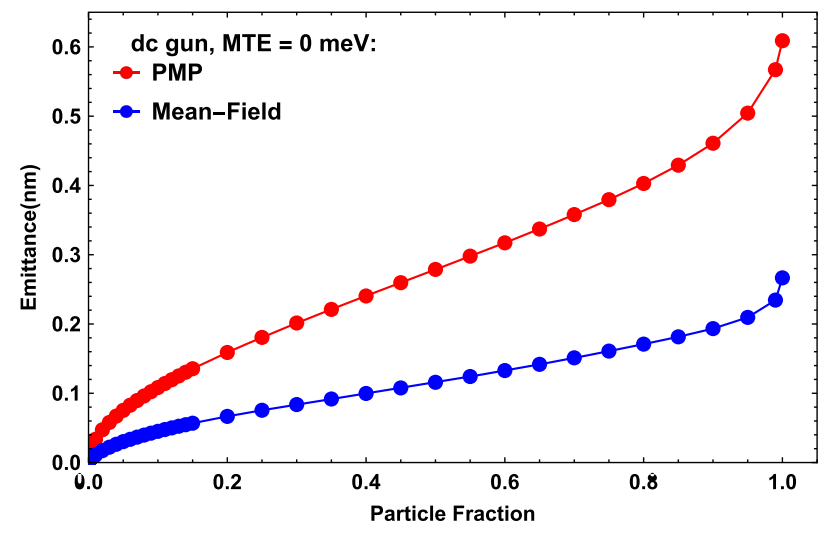

(a)

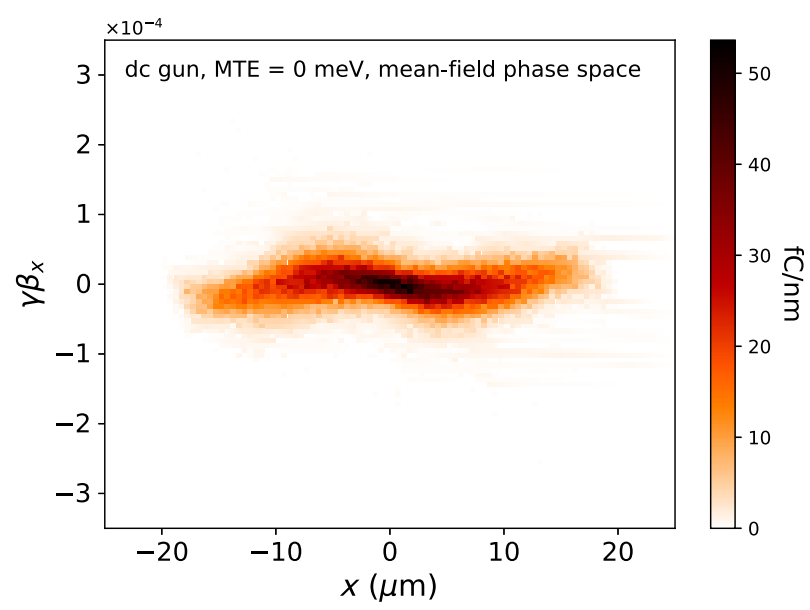

(c)

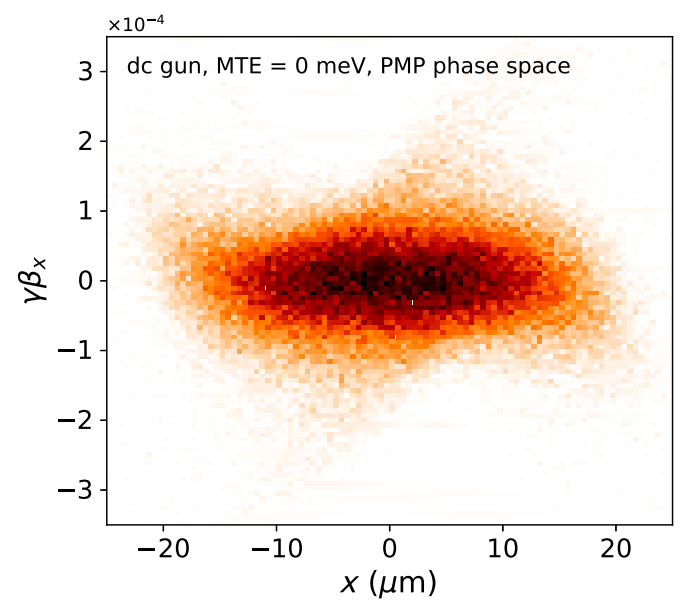

(e)

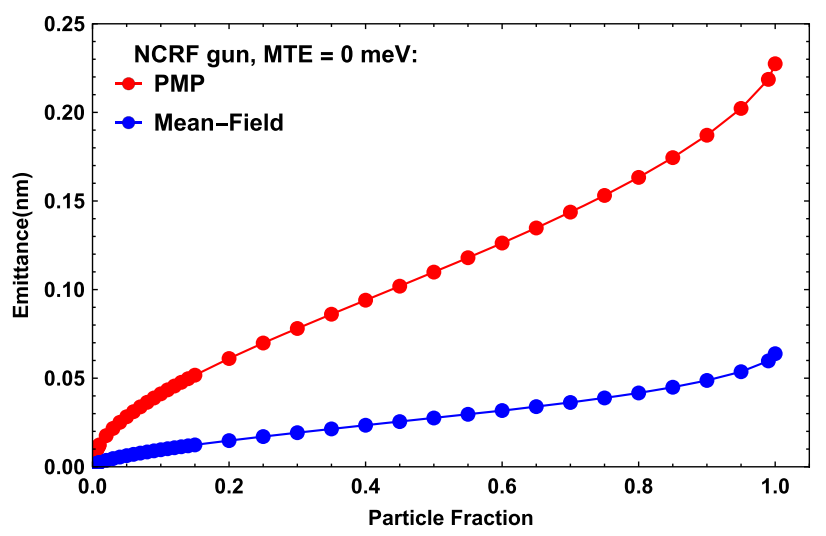

(b)

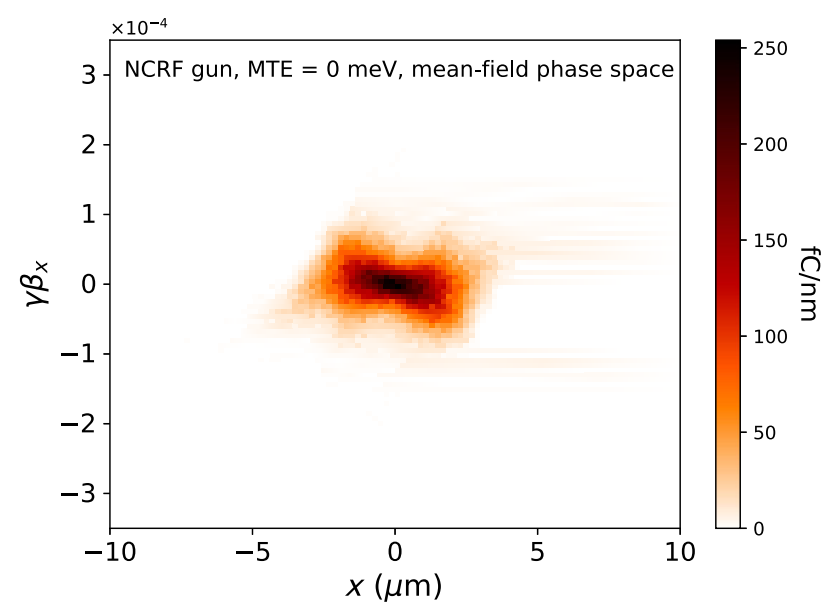

(d)

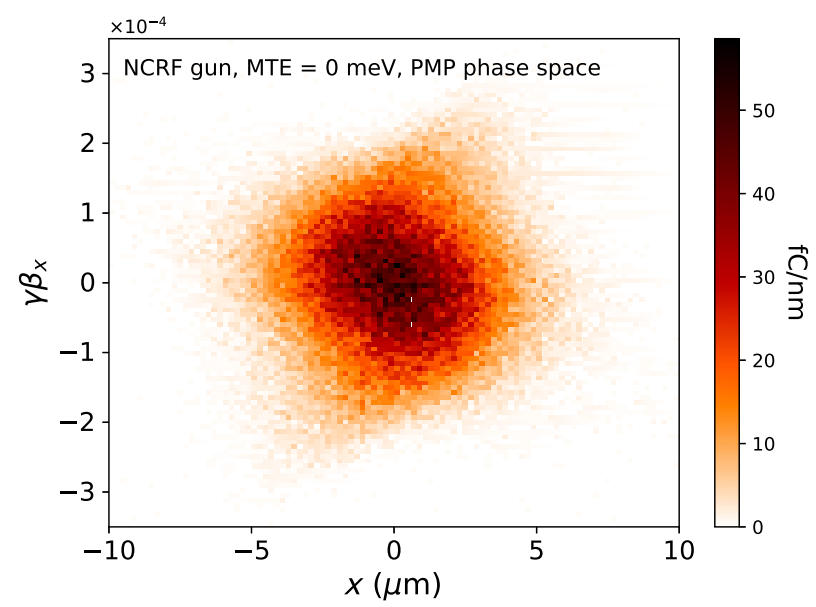

(f)

FIG. 7. Transverse normalized rms emittance vs particle fraction plots and phase space comparison between PMP and mean-field simulations of the two UED beam lines at the respective emittance minimum near the end of the beam lines. Subfigures (a), (c), and (e) correspond to the dc beam line and subfigures (b), (d), and (f) correspond to the NCRF beam line. Phase space portraits from the mean-field simulations are shown in subfigures (c) and (d) and for PMP simulations in (e) and (f) phase space portraits are shown with linear $x-p_{x}$ correlation removed. 


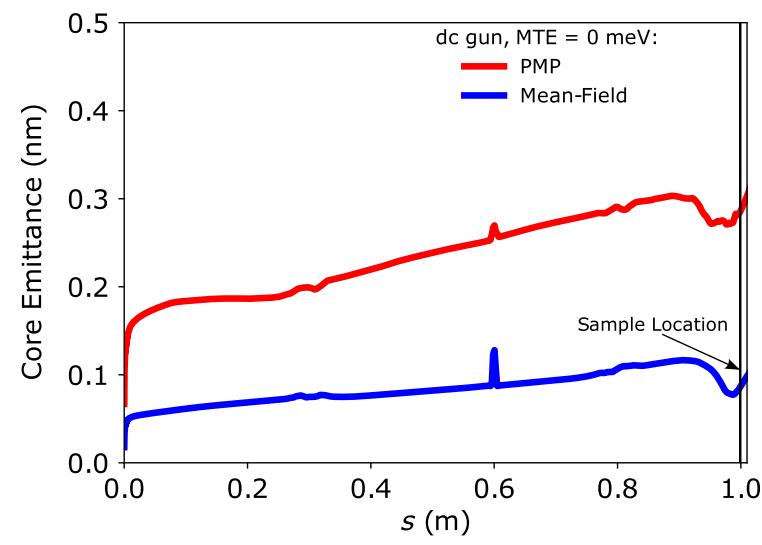

FIG. 8. Core emittance comparison between PMP and meanfield simulations of the dc UED beam line with $0 \mathrm{meV}$ MTE.

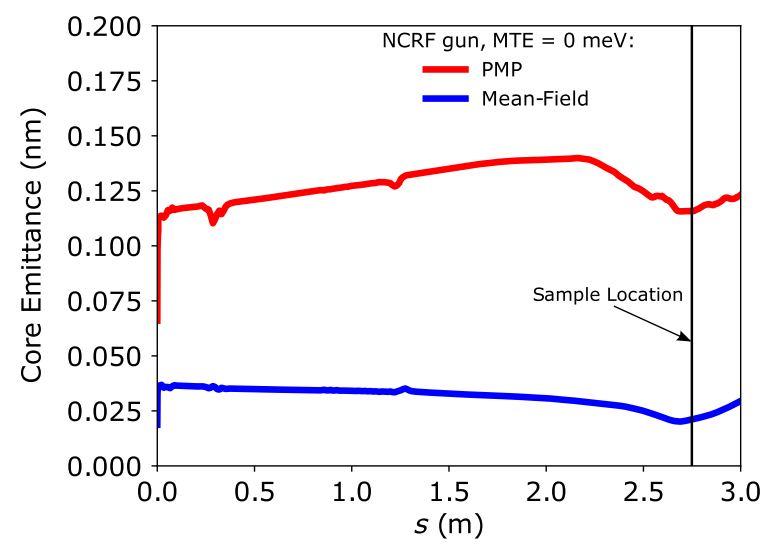

FIG. 9. Core emittance comparison between PMP and meanfield space charge for the NCRF UED beam line with 0 MTE.

particle is calculated. By making a histogram of these distances, we generate a plot of $g(r) \times \rho N \times 4 \pi r^{2} \Delta r$ as a function of $r$, where $N$ is the number of particles, $\rho$ is the bulk volume density of those particles, and $\Delta r$ is the bin size. A statistical uncertainty is assigned to each bin equal to the square root of the number of particles in the bin. Dividing out the $r^{2}$ term, $g(r)$ is found up to numerical prefactors.

The radial distribution function shortly after emission in the case of the NCRF UED beam line with $0 \mathrm{meV}$ MTE is shown in Fig. 10. For distances smaller than $1.5 \mu \mathrm{m}$, the radial distribution function in the case of point-to-point space charge decreases to 0 as expected, while $g(r)$ of

TABLE IV. Core emittance with $0 \mathrm{meV}$ MTE at sample.

\begin{tabular}{lcc}
\hline \hline Beam line & PMP $\epsilon_{c}(\mathrm{~nm})$ & Mean-field $\epsilon_{c}(\mathrm{~nm})$ \\
\hline $\mathrm{dc}$ & 0.28 & 0.08 \\
$\mathrm{NCRF}$ & 0.12 & 0.020 \\
\hline \hline
\end{tabular}

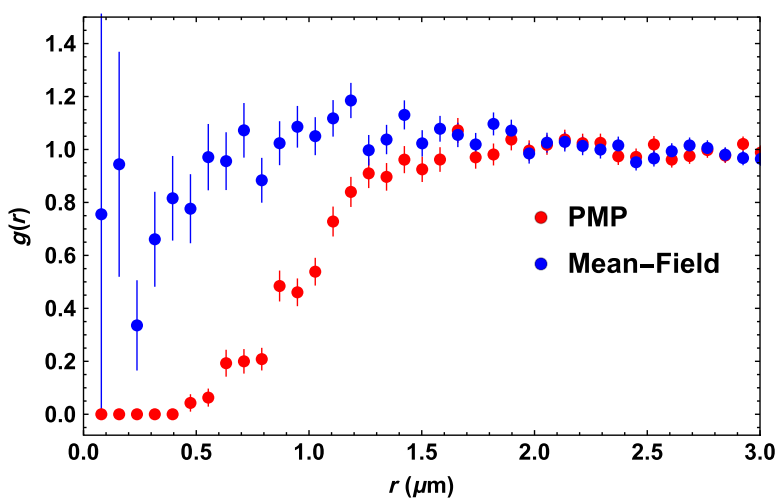

FIG. 10. Radial distribution function comparison between PMP and mean-field simulations of the NCRF UED beam line with $0 \mathrm{meV}$ MTE $\sim 3 \mathrm{~mm}$ away from the cathode. Only a small $r$ portion of the distribution is plotted to show the creation of the Coulomb hole when point-to-point space charge is used. For comparison, the distributions were normalized such that the mean of the radial distribution functions from 1.5 to $3.0 \mu \mathrm{m}$ is equal to 1 .

mean-field space charge does not. This same behavior can be seen for the dc beam line with an MTE of $0 \mathrm{meV}$.

\section{DIH calculation}

From the radial distribution function, the potential energy of a particle due to its surrounding particles can be calculated as

$$
E_{\text {potential }}=\int_{0}^{\infty} 4 \pi r^{2} \rho g(r) u(r) \mathrm{d} r
$$

where $\rho$ is the bulk volume density of the beam and $u(r)$ is the potential energy of two electrons particles separated by a distance $r$ [44]. Using Eq. (4), $E_{\mathrm{DIH}}$ can be calculated by finding the difference between the potential energy calculated via the radial distribution function in the point-topoint simulation and a calculation using the same radial distribution function where the Coulomb hole is artificially filled. Tests with stationary distributions which have known $E_{\text {DIH }}$ show that this estimation method is accurate to within $20 \%$, with discrepancies arising primarily due to the determination of the peak location of $g(r)$.

This energy difference would be $E_{\mathrm{DIH}}$ for all times after heating if the beam did not change in size throughout the simulation. Because the beam size changes, additional calculation is required to recover $E_{\mathrm{DIH}}$. If the beam changes in a self-similar way, such that its aspect ratio remains constant, the energy found through this subtraction is $E_{\mathrm{DIH}}$ multiplied by the ratio of the initial average interparticle distance to the current average interparticle distance. This can be seen by investigating the radial scaling of Eq. (4) noting that $\rho \propto 1 / r^{3}$ and $u(r) \propto 1 / r$. Thus by multiplying by the inverse of this factor, we can estimate $E_{\mathrm{DIH}}$ assuming DIH takes place very near the cathode. Further, it is clear 
the assumption of self-similarity is invalid if the beam deviates significantly from its initial aspect ratio, which can occur when space charge forces cause significant "blowout" (spatial [45] or longitudinal [46]) and near most beam waists.

The initial interparticle spacing and initial electron density require definition, as at $t=0$, no beam yet exists. To do this, we will approximate the beam as a uniform cylinder with equivalent rms sizes as at the cathode surface. Assuming a uniform acceleration over the small length and timescale the beam is being emitted from the cathode, the front of the beam will travel to a distance of $L=\frac{1}{2} a_{E_{0}} t^{2}$, where $a_{E_{0}}$ is the acceleration of an electron in a uniform electric field $E_{0}$, and $t$ is the difference in time between the first and last particle emitted. Approximating as a uniform distribution where $R=2 \sigma_{R}$ and $t=\sqrt{12} \sigma_{t}$, the volume of the beam can be found as

$$
V=\pi R^{2} L \approx \frac{24 \pi e E_{0}}{m c^{2}} \sigma_{x}^{2}\left(c \sigma_{t}\right)^{2} .
$$

Using this volume, an initial average interparticle distance can be found as $\rho \approx(V / N)^{1 / 3}$ and initial electron density is $n_{0} \approx(N / V)$.

A plot of the $E_{\mathrm{DIH}}$ estimate is shown in Fig. 11. There are three main features of this plot. First, there is an initial rise in $E_{\mathrm{DIH}}$ corresponding to the time it takes for the Coulomb hole to form, i.e., the inverse plasma oscillation frequency of the beam is

$$
\tau \approx 0.3 \frac{2 \pi}{\omega_{p}}=0.6 \pi\left(\frac{n_{0} e^{2}}{m \epsilon_{0}}\right)^{-1 / 2}
$$

where $n_{0}$ is the density of the electron beam [28]. After the initial rise there is a plateau. The mean value of this plateau is used for the value of $E_{\mathrm{DIH}}$ of the simulation and the standard deviation of these values is treated as an

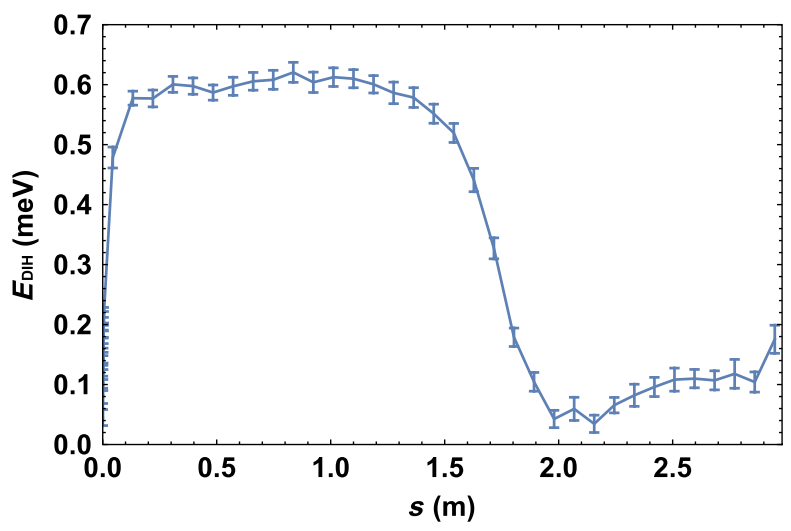

FIG. 11. Energy from disorder-induced heating as calculated from $g(r)$ in the NCRF beam line with 0 MTE and a smaller initial density of $10^{17} \mathrm{~m}^{-3}$. The density was reduced by increasing the initial radial size of the electron beam at the cathode.

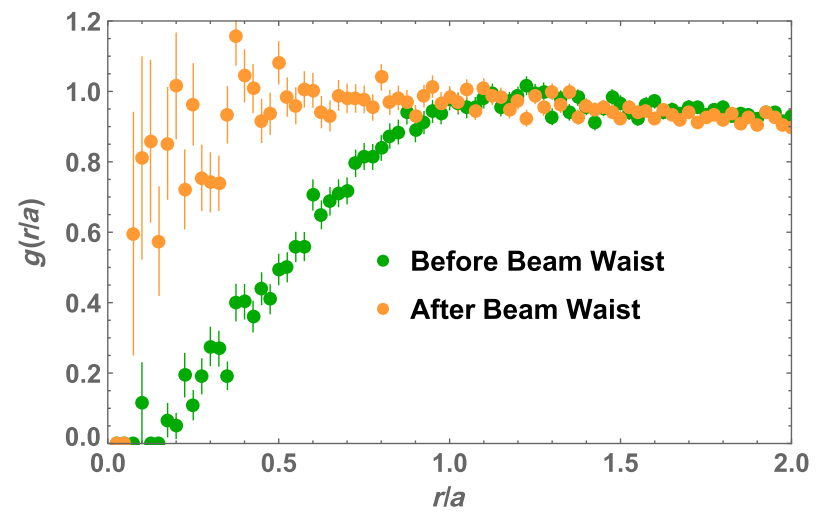

FIG. 12. Radial distribution function comparison between of the NCRF beam line with an MTE of $0 \mathrm{meV}$ and an initial density of $10^{17} \mathrm{~m}^{-3}$ before and after the beam waist. For comparison, the distances were normalized by the average interparticle distance, $a$, and the radial distribution functions, $g(r / a)$, were normalized such that $g(r / a=1.25)=1$.

uncertainty. The drop in $E_{\mathrm{DIH}}$ corresponds to the transverse focus of the beam. During this focusing, not only is the selfsimilarity assumption violated, there is another microscopic reorganization in which the Coulomb hole is filled. This is shown in Fig. 12. Please note that this downstream filling in the Coulomb hole does not have a significant impact on the core emittance of the beam anymore. This is because near the beam waist, the transverse temperature of the of the beam $m c^{2}\left(\epsilon / \sigma_{x}\right)^{2}$ is $\sim 12 \mathrm{meV}$ and the energy per particle required to fill the Coulomb hole is $\sim 0.3 \mathrm{meV}$.

\section{DIH density dependence}

For a stationary electron plasma with a starting temperature of zero, the energy released by DIH can be calculated via Eq. (2). To test the density dependence of Eq. (2) in the realistic, nonstationary case, this procedure was repeated on simulations of the same NCRF beam line with 0 MTE, while changing the radius of the inital beam in order to alter its density. The result is shown in Fig. 13.

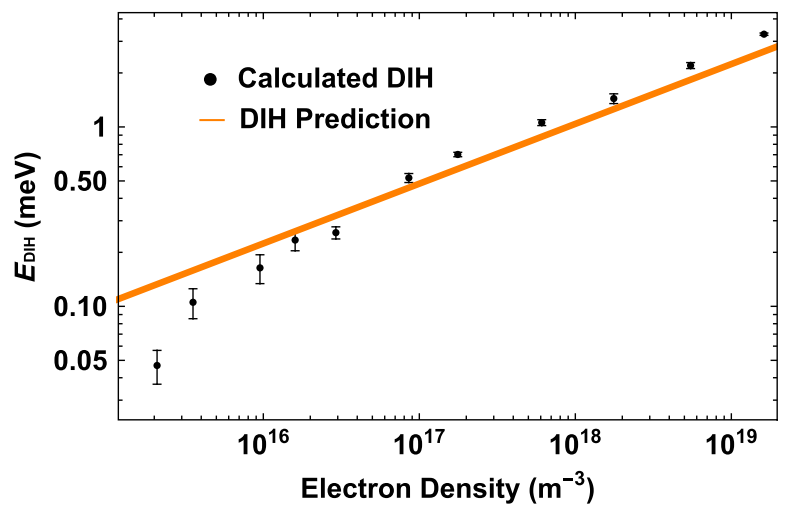

FIG. 13. Disorder-induced heating as calculated from Eq. (2) compared to the result calculated from simulation. 
For densities of $10^{16} \mathrm{~m}^{-3}$ and above, the simulation results agree with the simple stationary theory within a factor of 2. At a density near $10^{15} \mathrm{~m}^{-1 / 3}$, the timescale for heating is $\sim 1 \mathrm{~ns}$, which is approximately the time it takes the beam to enter the first solenoid. Because the beam has had time to change significantly in size and shape, there is no reason to expect that the approximations used in calculating $E_{\mathrm{DIH}}$ remain true, thus it will be ignored in the following analysis. We find a that $E_{\mathrm{DIH}}$ in our simulations scales with density to the power $0.39 \pm 0.02$, close the value of $1 / 3$ in Eq. (2).

\section{E. Core emittance and rms emittance contributions from DIH}

From the calculated values of $E_{\mathrm{DIH}}$, we can estimate the expected increase in the core emittance, and rms transverse emittance from DIH alone. This will help determine to what extent the Coulomb hole formation determines the growth in core and rms emittance.

For the core emittance, starting from Eq. (3), the density in $x-p_{x}$ space at the transverse origin can be calculated assuming a cylindrical beam shape and a Gaussian momentum distribution,

$$
\epsilon_{c}=\frac{\sqrt{2 \pi}}{4} \sigma_{x} \sigma_{p_{x}}
$$

The initial spread of momenta can be written in terms of the MTE in the standard way,

$$
\sigma_{p_{x}}=\sqrt{\frac{\mathrm{MTE}}{m c^{2}}} .
$$

For the presented simulations, the initial MTE is 0 , but some of the DIH energy will be released in the transverse phase space, resulting in a nonzero momentum spread. In general, the distribution of the heating will depend on the shape of the beam. However, in the case that the average interparticle distance is much less than the smallest length scale of the beam, the bulk heating effect will dominate and the edge effects can be ignored. In this approximation, the heating is isotropic and $2 / 3$ of the $E_{\mathrm{DIH}}$ will contribute to the MTE of the beam. Assuming that the initial and DIH contributions can be added in quadrature, the core emittance becomes approximately,

$$
\epsilon_{c} \approx \frac{\sqrt{2 \pi}}{4} \sigma_{x} \sqrt{\frac{\mathrm{MTE}+\frac{2}{3} E_{\mathrm{DIH}}}{m c^{2}}} .
$$

In the case of the dc (NCRF) beam line with $0 \mathrm{meV}$ MTE, the initial transverse size of the beam is $8.2 \mu \mathrm{m}$ $(2.6 \mu \mathrm{m})$ and the energy from DIH is $0.65 \mathrm{meV}(1.4 \mathrm{meV})$, so the resulting core emittance due to $E_{\mathrm{DIH}}$ is $0.18 \mathrm{~nm}$ $(0.070 \mathrm{~nm})$.
To compare these results to those found in the previous sections, we must take into account the increase of the core emittance in the mean-field space charge simulation from 0 , which is an effect of finite sampling. To do this, we will assume the effects of point-to-point space charge can be added in quadrature to the mean-field core emittance, analogously to Eq. (9) and as is valid for independent rms emittance contributions. With this assumption, the core emittance contribution of point-to-point space charge, $\epsilon_{c, P 2 P}$, can be found through a quadrature subtraction of the mean-field core emittance from the PMP core emittance. For the dc (NCRF) gun beam line, $\epsilon_{c, P 2 P}$ at the sample is $0.27 \mathrm{~nm}(0.12 \mathrm{~nm}) .67 \%$ of $\epsilon_{c, P 2 P}$ at the sample in the dc case is explained by $E_{\mathrm{DIH}}(0.27 \mathrm{~nm}$ vs $0.18 \mathrm{~nm})$, and in the rf case, $58 \%$ of $\epsilon_{c, P 2 P}$ at the sample is determined by $E_{\text {DIH }}(0.12 \mathrm{~nm}$ vs $0.070 \mathrm{~nm})$.

For the rms transverse emittance contribution, we will use the intrinsic emittance of the beam line [6],

$$
\epsilon_{i}=\sigma_{x} \sqrt{\frac{\mathrm{MTE}}{m c^{2}}} .
$$

As with the core emittance, adding in $2 / 3$ of the DIH energy, a modification is made to the intrinsic emittance equation,

$$
\epsilon_{i} \approx \sigma_{x} \sqrt{\frac{\mathrm{MTE}+\frac{2}{3} E_{\mathrm{DIH}}}{m c^{2}}} .
$$

In the dc (NCRF) beam line, the intrinsic $90 \%$ transverse emittance including $E_{\mathrm{DIH}}$ is $0.27 \mathrm{~nm}(0.11 \mathrm{~nm})$. We will compare this to the quadrature subtraction of the $90 \%$ transverse emittance of the mean-field simulation from the PMP simulation, which we will call $\epsilon_{P 2 P}$. At the emittance minimum, $\epsilon_{P 2 P}$ in the dc (NCRF) beam line is $0.40 \mathrm{~nm}$ $(0.18 \mathrm{~nm})$. The intrinsic emittance contribution from DIH in the dc (NCRF) beam line thus accounts for $68 \%$ (61\%) of $\epsilon_{P 2 P}$. The remaining difference can be attributed to the effect of large angle scatters which kick particles far from the beam center, seen as the tails of the emittance fraction curve in Fig. 7(a).

\section{CONCLUSION}

In this work, we have shown that as photoemitted electron beam temperatures are made ever smaller, the effects of the point like nature of the Coulomb interaction become crucial to understanding photoinjector beam dynamics. We have introduced and benchmarked a simple method to compute the image force in a point-to-point beam dynamics simulations free of divergences and additional tuning parameters. Using this method, we have quantified Coulomb scattering effects on the beam phase space density in two UED beam line archetypes. Using a photocathode with zero intrinsic emittance, the emittance of 
the beam in both the rf and dc UED beam lines studied was larger by a factor of at least 2, see Figs. 4(b) and 5(b), and the core emittance is larger by a factor of at least 3 , see Figs. 8 and 9, when compared to simulation on the same beam line but assuming mean-field space charge. In addition, the energy released by DIH was calculated using the radial distribution function, and the heating was found to scale with the density to the power of $0.39 \pm 0.03$, close to the a simple theoretical estimate of $1 / 3$, and was shown to be the dominant effect in both core and $90 \% \mathrm{rms}$ emittance growth. We found DIH contributed to the emittance of the beam lines studied as shown in Eq. (11).

\section{ACKNOWLEDGMENTS}

This work was supported by the Center for Bright Beams, Grant No. NSF PHY-1549132.

\section{APPENDIX A: WARM BEAM COMPARISON}

In this section, the same dc and NCRF UED beam lines were simulated using a initial beam MTE of $150 \mathrm{meV}$. By doing so, we aim to show that the PMP and mean-field methods converge to the same result for a warm photocathode, and that Debye screen effectively mitigates pointto-point effects.

In Figs. 14(a) and 15(a), the spot size evolution is shown for the dc and NCRF UED beam lines, respectively, each with $150 \mathrm{meV}$ MTE. At this higher MTE, the difference between the point-to-point and mean-field spot size has been significantly reduced. At the beams maximal size in the first solenoid, the deviation has been reduced from around 2 to $0.7 \mu \mathrm{m}$ in the dc beam line and 1.2 to $0.5 \mu \mathrm{m}$ in the NCRF beam line.

The evolution of the transverse rms emittance for the dc and NCRF beam lines is shown in Figs. 14(b) and 15(b), respectively. With an MTE of $150 \mathrm{meV}$, there is no significant deviation in the transverse rms emittance between the implementations of space charge as observed at $0 \mathrm{meV}$. This validates that phtocathodes with high emission temperatures can be successfully modeled without consideration of point-to-point space charge effects.

The evolution of the core emittance at an MTE of $150 \mathrm{meV}$ is shown in Figs. 14(c) and 15(c) for the dc and NCRF UED beam lines, respectively. Outside of fluctuations near the solenoids the core emittance in all simulations are approximately constant. No significant difference exists between the core emittance between point-to-point and mean-field simulations at $150 \mathrm{meV}$.

\section{APPENDIX B: MODIFIED IMAGE CHARGE METHOD}

The majority of this manuscript employs a mean-field model of the image force. In this Appendix, we show this to be a valid approximation. To do this, we analytically investigate a pointlike image model and compare it to PMP.

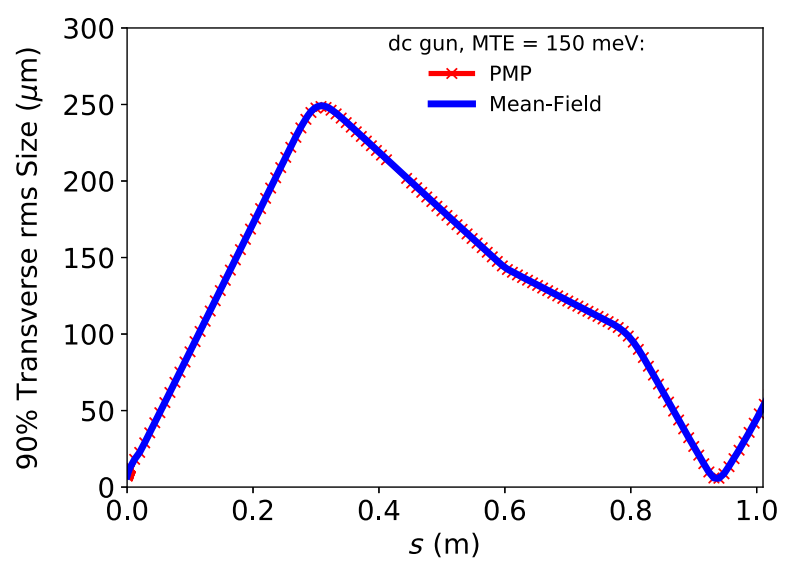

(a)

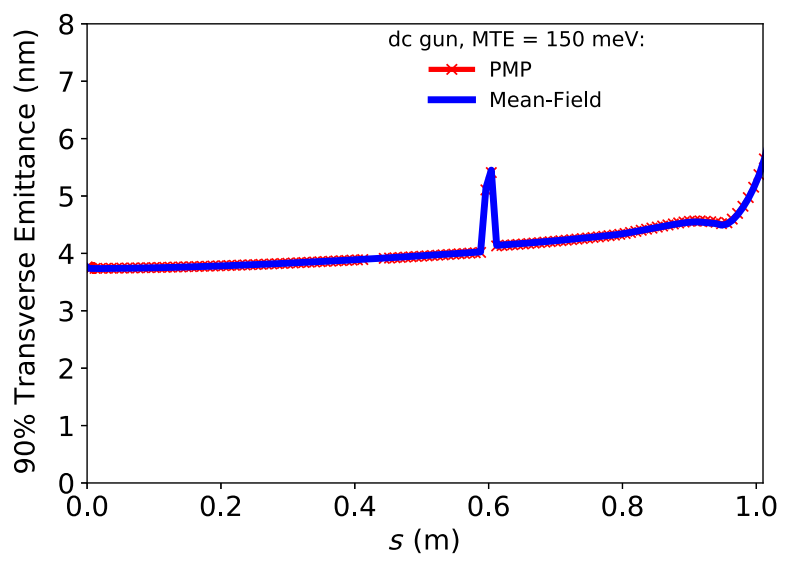

(b)

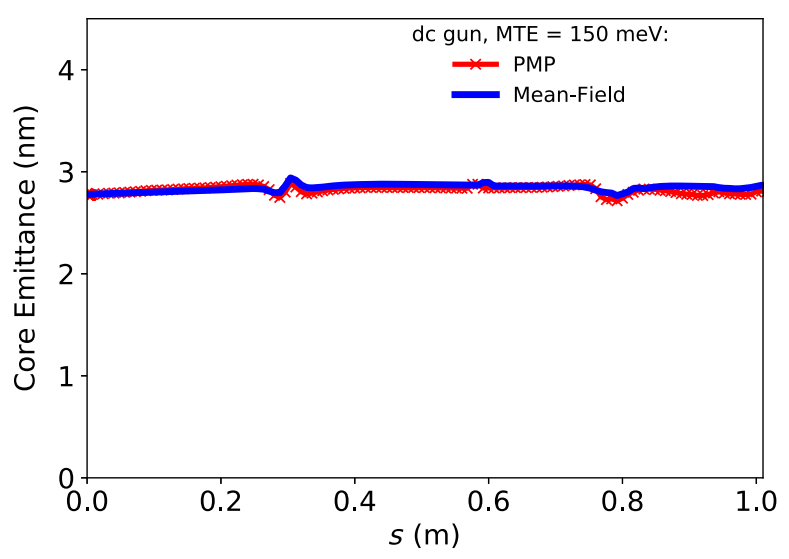

(c)

FIG. 14. (a) Spot size, (b) transverse normalized rms emittance, and (c) core emittance comparison between the PMP method and mean-field space charge simulations of the dc UED beamline with $150 \mathrm{meV}$ MTE.

We first aim to show that for particles much closer to the cathode compared to the average interparticle distance, the Coulombic repulsion force will be predominantly 


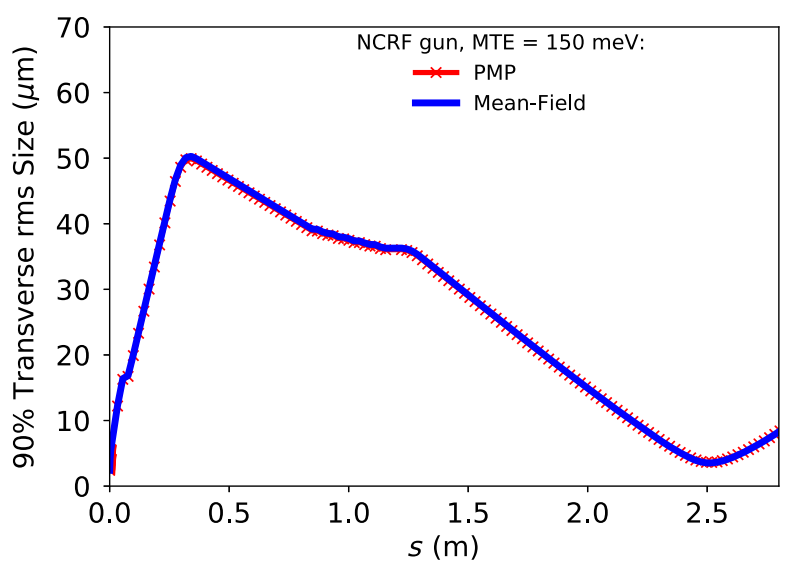

(a)

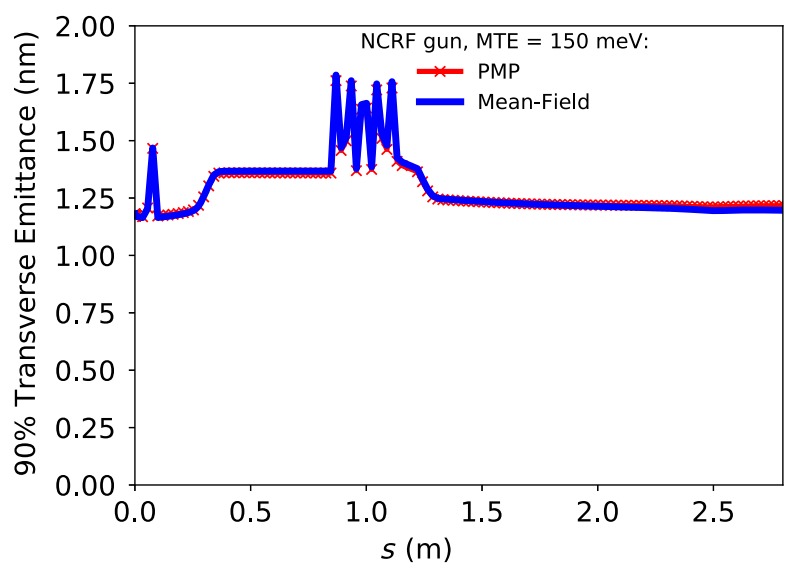

(b)

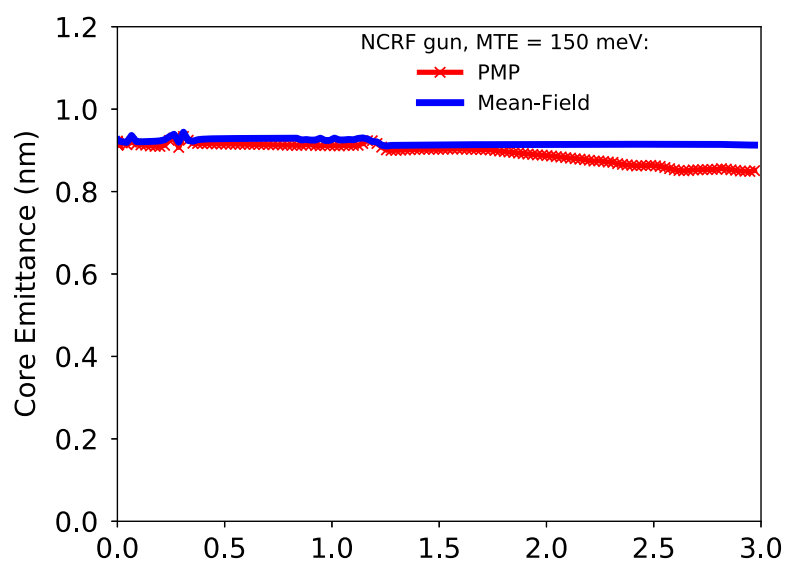

(c)

FIG. 15. (a) Spot size, (b) transverse normalized rms emittance, and (c) core emittance comparison between the PMP method and mean-field space charge simulations of the NCRF UED beamline with $150 \mathrm{meV}$ MTE.

longitudinal, as the transverse fields from other charges and their images will largely cancel.

The transverse electric field from an electron a distance $d$ away from an infinite conducting plane and its image charge in cylindrical coordinates is given by,

$E_{r}=\frac{1}{4 \pi \epsilon_{0}}\left(\frac{-e r}{\left(r^{2}+(z-d)^{2}\right)^{3 / 2}}+\frac{e r}{\left(r^{2}+(z+d)^{2}\right)^{3 / 2}}\right)$.

We first will consider the effects of particles far away from the cathode on particles which are recently emitted. At a position much closer to the cathode $(z \ll d)$, this expression is approximately,

$$
E_{r} \approx \frac{1}{4 \pi \epsilon_{0}} \frac{-3 e z r d}{2\left(r^{2}+d^{2}\right)^{5 / 2}} .
$$

We compare this to the longitudinal field in cylindrical coordinates,

$E_{z}=\frac{1}{4 \pi \epsilon_{0}}\left(\frac{-e(z-d)}{\left(r^{2}+(z-d)^{2}\right)^{3 / 2}}+\frac{e(z+d)}{\left(r^{2}+(z+d)^{2}\right)^{3 / 2}}\right)$.

In the same limit, $z \ll d$,

$$
E_{z} \approx \frac{1}{4 \pi \epsilon_{0}} \frac{2 e d}{\left(r^{2}+d^{2}\right)^{3 / 2}} .
$$

The magnitude of the ratio of the transverse and longitudinal electric fields in this limit of $z \ll d$ is

$$
\left|\frac{E_{r}}{E_{z}}\right| \approx \frac{3 z r}{4\left(r^{2}+d^{2}\right)}
$$

This ratio tends to 0 for small or large $r$ and has a maximum at $r=d$ of

$$
\left|\frac{E_{r}}{E_{z}}\right|_{\max } \approx \frac{3 z}{8 d} .
$$

Because $z \ll d$ the transverse electric field will be much smaller than the longitudinal field, and thus proving the Coulombic repulsion force will be predominantly longitudinal.

For an electron beam with an average interparticle distance $a$, this will apply as long as $a \gg d$. Therefore, for particles that have just been emitted, if we are to model the effects of the cathode and to avoid divergent fields for small $z$, we can ignore transverse effects, and only need to find the time it takes for a particle to travel a longitudinal distance on the order of the interparticle distance, and its energy at that point.

From dynamical image charge theory, a semiclassical image potential can be computed [33], in the approximation that the electron has no velocity parallel to the conducting surface, the potential energy $\mathrm{V}$ is 


$$
\begin{aligned}
V(v, z) & =-\frac{1}{4 \pi \epsilon_{0}} \frac{e^{2} \omega_{p}^{2}}{4 v \omega_{s}} f\left(\frac{2 z \omega_{s}}{v}\right), \\
f(x) & =\int_{0}^{\infty} \frac{e^{-\alpha x}}{1+\alpha^{2}} d \alpha
\end{aligned}
$$

where $\omega_{p}$ is the plasma frequency of the material, $\omega_{p}^{2}=2 \omega_{s}^{2}, v$ is the velocity of the electron, and $z$ is its distance from the cathode.

The energy of an electron moving at a velocity $v$ a distance $z$ from the cathode with an applied electric field $E_{z}$ is thus,

$$
E=\frac{m v^{2}}{2}-\frac{1}{4 \pi \epsilon_{0}} \frac{e^{2} \omega_{p}^{2}}{4 v \omega_{s}} f\left(\frac{2 z \omega_{s}}{v}\right)-e E_{z} z
$$

The applied electric field will consist of the field from the gun as well as an approximation to the longitudinal effects of other particles. The field from the particles in front of a given particle will be approximated as a uniformly charged cylinder with a transverse size, $R$, equal to that of the beam, and longitudinal size, $L$, equal to the initial bunch length multiplied by the beam fraction that has left the cathode previously. The image charge contribution from other particles will be approximated as a positively charged cylinder with equal dimensions placed directly behind the particle. With this, the equation for the applied electric field on the $j$ th particle emitted is

$E_{z}(j)=E_{\mathrm{gun}}(z)+\frac{e(j-1)}{\pi R^{2} \epsilon_{0}}\left(1+\frac{R}{L(j)}-\sqrt{1+\frac{R^{2}}{L(j)^{2}}}\right)$

where $L(j)$ is the length of the bunch in front of the $j$ th particle.

These equations can be self-consistently solved to find the velocity and potential at any longitudinal position for a given energy $E$. Calculating the velocity at several locations near the cathode, the time it takes to reach a given distance can be calculated as well. Because the transverse effects of space charge can be ignored in this regime, the external transverse field near the cathode can be used to approximate the transverse position of particle at a later distance, although we find this effect to be insignificant for the cases considered in this manuscript.

With this information, the beam can be initialized in simulation at a position away from the cathode divergence and thereafter can be modeled using a standard, pointlike image charge method. As long as the position is chosen to be sufficiently far from the cathode and less than the average particle distance, the resulting simulation should not depend heavily on the starting distance choice.

Through this analysis, we have shown that the image charges have little effect on the transverse dynamics of

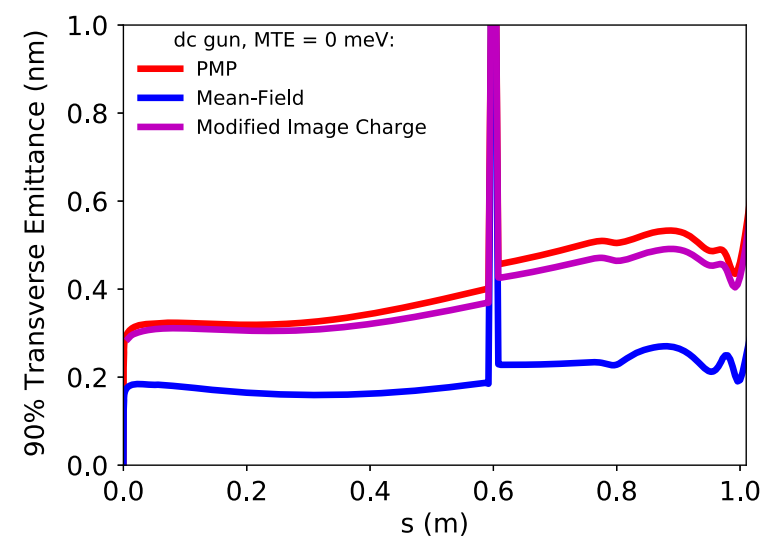

FIG. 16. Transverse normalized rms emittance comparison for point-to-point, mean-field, and modified image charge simulations of the dc UED beam line with $0 \mathrm{meV}$ MTE.

particles until a distance away from the cathode on the order of the average interparticle distance. Because pointto-point effects are most important for interactions at a distance less than the average interparticle distance, a mean-field implementation of the image charge, such as is done through the PMP method used in this paper, should produce correct results as long as the energy modulation of the produced particles by their images is correct.

In Figs. 16 and 17, the $0 \mathrm{meV}$ MTE transverse normalized rms emittance results for the DC and NCRF beam line are shown, respectively, now including results from the modified image charge method discussed in this Appendix. $E$ was chosen such that the minimum kinetic energy of an electron was $5 \mathrm{meV}$, on the order of the smallest MTE measured today. The particles were started at a distance $0.5 \mu \mathrm{m}$ away from the cathode. Deviations in the emittance from the PMP method are less than $10 \%$ throughout the simulations.

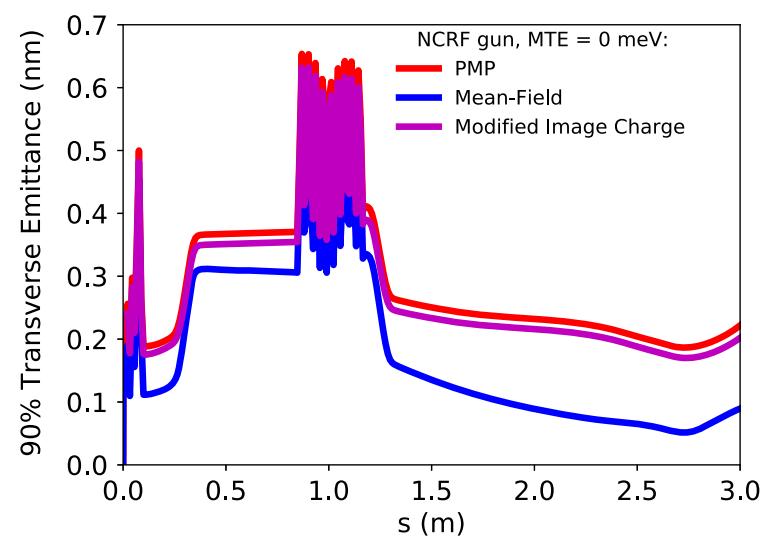

FIG. 17. Transverse normalized rms emittance comparison for point-to-point, mean-field, and modified image charge simulations for the NCRF UED beam line with $0 \mathrm{meV}$ MTE. 
[1] P. Musumeci, J. Giner Navarro, J. B. Rosenzweig, L. Cultrera, I. Bazarov, J. Maxson, S. Karkare, and H. Padmore, Advances in bright electron sources, Nucl. Instrum. Methods Phys. Res., Sect. A 907, 209 (2018).

[2] S. M. Gruner, D. Bilderback, I. Bazarov, K. Finkelstein, G. Krafft, L. Merminga, H. Padamsee, Q. Shen, C. Sinclair, and M. Tigner, Energy recovery linacs as synchrotron radiation sources (invited), Rev. Sci. Instrum. 73, 1402 (2002).

[3] P. Emma et al., First lasing and operation of an ångstromwavelength free-electron laser, Nat. Photonics 4, 641 (2010).

[4] T. van Oudheusden, E. F. de Jong, S. B. van der Geer, W. P. E. M. Op 't Root, O. J. Luiten, and B. J. Siwick, Electron source concept for single-shot sub-100 fs electron diffraction in the $100 \mathrm{kev}$ range, J. Appl. Phys. 102, 093501 (2007).

[5] I. V. Bazarov, B. M. Dunham, Y. Li, X. Liu, D. G. Ouzounov, C. K. Sinclair, F. Hannon, and T. Miyajima, Thermal emittance and response time measurements of negative electron affinity photocathodes, J. Appl. Phys. 103, 054901 (2008).

[6] H. Lee, S. Karkare, L. Cultrera, A. Kim, and I. V. Bazarov, Review and demonstration of ultra-low-emittance photocathode measurements, Rev. Sci. Instrum. 86, 073309 (2015).

[7] B. E. Carlsten, Space-charge-induced emittance compensation in high-brightness photoinjectors, Part. Accel. 49, 27 (1995).

[8] L. Serafini and J. B. Rosenzweig, Envelope analysis of intense relativistic quasilaminar beams in rf photoinjectors: $\mathrm{mA}$ theory of emittance compensation, Phys. Rev. E 55, 7565 (1997).

[9] C. M. Pierce, M. B. Andorf, E. Lu, C. Gulliford, I. V. Bazarov, J. M. Maxson, M. Gordon, Y.-K. Kim, N. P. Norvell, B. M. Dunham, and T. O. Raubenheimer, Low intrinsic emittance in modern photoinjector brightness, Phys. Rev. Accel. Beams 23, 070101 (2020).

[10] S. P. Weathersby et al., Mega-electron-volt ultrafast electron diffraction at slac national accelerator laboratory, Rev. Sci. Instrum. 86, 073702 (2015).

[11] Y. Ding et al., Measurements and Simulations of Ultralow Emittance and Ultrashort Electron Beams in the Linac Coherent Light Source, Phys. Rev. Lett. 102, 254801 (2009).

[12] M. Altarelli et al., XFEL: The European X-ray free-electron laser, Technical Report No. 7, 2006.

[13] J. Feng, J. Nasiatka, W. Wan, S. Karkare, J. Smedley, and H. A. Padmore, Thermal limit to the intrinsic emittance from metal photocathodes, Appl. Phys. Lett. 107, 134101 (2015).

[14] S. Karkare, G. Adhikari, W. A. Schroeder, J. K. Nangoi, T. Arias, J. Maxson, and H. Padmore, Ultracold Electrons via Near-Threshold Photoemission from Single-Crystal $\mathrm{Cu}$ (100), Phys. Rev. Lett. 125, 054801 (2020).

[15] M. Reiser, Linear Beam Optics with Space Charge: Sections 4.1-4.4, ( John Wiley \& Sons Ltd, New York, 2008), Chap. 4, pp. 163-232, ISBN 9783527622047.

[16] A. J. McCulloch, D. V. Sheludko, M. Junker, and R. E. Scholten, High-coherence picosecond electron bunches from cold atoms, Nat. Commun. 4, 1692 (2013).
[17] D. Murphy, R. Speirs, D. Sheludko, C. Putkunz, A. Mcculloch, B. Sparkes, and R. Scholten, Detailed observation of space-charge dynamics using ultracold ion bunches, Nat. Commun. 5, 4489 (2014).

[18] B. J. Claessens, S. B. van der Geer, G. Taban, E. J. D. Vredenbregt, and O. J. Luiten, Ultracold Electron Source, Phys. Rev. Lett. 95, 164801 (2005).

[19] J. G. H. Franssen, T.C.H. de Raadt, M. A. W. van Ninhuijs, and O.J. Luiten, Compact ultracold electron source based on a grating magneto-optical trap, Phys. Rev. Accel. Beams 22, 023401 (2019).

[20] S. B. van der Geer, M. J. de Loos, E. J. D. Vredenbregt, and O. J. Luiten, Ultracold electron source for single-shot, ultrafast electron diffraction, Microsc. Microanal. 15, 282 (2009).

[21] J. G. H. Franssen, T. L. I. Frankort, E. J. D. Vredenbregt, and O. J. Luiten, Pulse length of ultracold electron bunches extracted from a laser cooled gas, Struct. Dyn. 4, 044010 (2017).

[22] W. J. Engelen, E. P. Smakman, D. J. Bakker, O. J. Luiten, and E.J.D. Vredenbregt, Effective temperature of an ultracold electron source based on near-threshold photoionization, Ultramicroscopy 136, 73 (2014).

[23] B. J. Claessens, M. P. Reijnders, G. Taban, O. J. Luiten, and E. J. D. Vredenbregt, Cold electron and ion beams generated from trapped atoms, Phys. Plasmas 14, 093101 (2007).

[24] J. Barnes and P. Hut, A hierarchical o(n $\log n$ ) forcecalculation algorithm, Nature (London) 324, 446 (1986).

[25] L. Greengard and V. Rokhlin, A fast algorithm for particle simulations, J. Comput. Phys. 73, 325 (1987).

[26] G. Sciaini and R. J.D. Miller, Femtosecond electron diffraction: Heralding the era of atomically resolved dynamics, Rep. Prog. Phys. 74, 096101 (2011).

[27] D. O. Gericke and M. S. Murillo, Disorder-induced heating of ultracold plasmas, Contrib. Plasma Phys. 43, 298 (2003).

[28] J. Maxson, I. Bazarov, C. Coleman-Smith, W. Wan, and H. Padmore, Fundamental photoemission brightness limit from disorder induced heating, New J. Phys. 15, 103024 (2013).

[29] D. H.E. Dubin and T. M. O'Neil, Trapped nonneutral plasmas, liquids, and crystals (the thermal equilibrium states), Rev. Mod. Phys. 71, 87 (1999).

[30] S. Chandrasekhar, Stochastic problems in physics and astronomy, Rev. Mod. Phys. 15, 1 (1943).

[31] Pulsar website for GPT. http://www.pulsar.nl/gpt/, 2011.

[32] S. B. van der Geer, O. J. Luiten, M. J. de Loos, U. van Rienen, and G. Pöplau, A fast 3d multigrid based spacecharge routine in the gpt code, in Proceedings of the European Particle Accel. Conf., Paris, France (2002), p. 1831.

[33] R. Ray and G. D. Mahan, Dynamical image charge theory, Phys. Lett. 42A, 301 (1972).

[34] G. H. Jansen, Coulomb interactions in particle beams, Nucl. Instrum. Methods Phys. Res., Sect. A 298, 496 (1990), ISSN .

[35] C. Gulliford, A. Bartnik, and I. Bazarov, Multiobjective optimizations of a novel cryocooled dc gun based ultrafast electron diffraction beam line, Phys. Rev. Accel. Beams 19, 093402 (2016). 
[36] C. Gulliford, A. Bartnik, I. Bazarov, and J. Maxson, Multiobjective optimization design of an $\mathrm{rf}$ gun based electron diffraction beam line, Phys. Rev. Accel. Beams 20, 033401 (2017).

[37] H. Lee, X. Liu, L. Cultrera, B. Dunham, V. O. Kostroun, and I. V. Bazarov, A cryogenically cooled high voltage dc photoemission electron source, Rev. Sci. Instrum. 89, 083303 (2018).

[38] P. L. E. M. Pasmans, G. B. van den Ham, S. F. P. Dal Conte, S. B. van der Geer, and O. J. Luiten, Microwave tm010 cavities as versatile $4 \mathrm{~d}$ electron optical elements, Ultramicroscopy 127, 19 (2013).

[39] D. T. Palmer, R. H. Miller, H. Winick, X. J. Wang, K. Batchelor, M.H. Woodle, and I. Ben-Zvi, Microwave measurements and beam dynamics simulations of the BNL/SLAC/UCLA emittance-compensated 1.6-cell photocathode rf gun, in Electron-Beam Sources and ChargedParticle Optics, edited by E. Munro and H. P. Freund (International Society for Optics and Photonics, SPIE, 1995), Vol. 2522, pp. 514-526, doi: https://doi.org/ $10.1117 / 12.221609$.
[40] R. B. Neal, The Stanford Two-Mile Accelerator, edited by W. A. Be (W. A. Benjamin Inc., New York, 1968), Vol. 1.

[41] I. V. Bazarov, Synchrotron radiation representation in phase space, Phys. Rev. ST Accel. Beams 15, 050703 (2012).

[42] D. Chandler, Introduction to Modern Statistical Mechanics (Oxford University Press, New York, 1987).

[43] E. J. Baerends and M. A. Buijse, Density Functional Theory of Molecules, Clusters, and Solids, Vol. 12 of Understanding Chemical Reactivity (Springer, Dordrecht, 1996).

[44] F. Mandl, Statistical Physics, 2nd ed. (Wiley, New York, 2013).

[45] D. Filippetto, P. Musumeci, M. Zolotorev, and G. Stupakov, Maximum current density and beam brightness achievable by laser-driven electron sources, Phys. Rev. ST Accel. Beams 17, 024201 (2014).

[46] P. Musumeci, J. T. Moody, R. J. England, J. B. Rosenzweig, and T. Tran, Experimental Generation and Characterization of Uniformly Filled Ellipsoidal Electron-Beam Distributions, Phys. Rev. Lett. 100, 244801 (2008). 